J. Asiat. Soc. Bangladesh, Sci. 40(2): 259-270, December 2014

\title{
HYDROPHYTES IN THE HIGH BARIND TRACT: DIVERSITY STATUS, THREATS AND CONSERVATION
}

\author{
SYEDA SALMA BEGUM \\ Ministry of Road Transport and Bridges, Road Transport and Highways Division, Bangladesh \\ Secretariat, Dhaka-1000, Bangladesh.
}

\begin{abstract}
A survey was conducted to assess the diversity of hydrophytes in some selected locations including Kharoil Beel (Mohanpur), Beel Kumari (Tanore) and Jobai Beel (Sapahar) situated in High Barind Tract, Rajshahi, Bangladesh from June 2005 to May 2008. Among them Jobai Beel is the largest, Beel Kumari is medium and Kharoil Beel is small in size. At experimental areas a total of 151 plant species and 90 genera distributed in 44 families was identified. From the result, diversity status of the study areas, the values ranged between 0.94-0.97 in Simpson's index and 3.55-4.13 in Shannon's index. Diversity status of three study areas shows richness. Sequence of richness is as follows- Kharoil Beel $>$ Beel Kumari > Jobai Beel. It is also evident that these wetiands are disturbed by anthropogenic activities. Some of the species have become threatened, some have become rare. Conservation efforts and awareness building are perquisite for the sustainability of these wetlands.
\end{abstract}

Key words: High barind tract, Hydrophytes, Diversity, Threats and conservation

\section{Introduction}

More than two thirds of Bangladesh may be classified as wetland according to the definition enunciated in the Ramasar Convention (Khan 1993). According to Ramasar Convention biologically and physically IUCN has identified a total of 39 categories of wetlands of which 30 are natural wetlands and nine man-made (Dugan 1990). Wetlands, have self sustained ecosystem which play a significant role for the formation of a wide array of biodiversity system, including diversified flora and fauna. Actually hydrophytes and wetland plants are defined in various ways. But presently broader and acceptable definition given by Cook (1996) is as- 'All pteridophytes and spermatophytes whose phtosynthetically active parts are permanently or at least for several months of each year part or whole submerged with water or which float in the surface of water' "and regarding the wetlands plants stated, wetland plants are those which grows in places where inundation must occur for at least fourteen days and saturation for at least sixty consecutive days. Prain (1903), Biswas and Caldar (1937), Cook (1996), Subramanyam (1962) etc. are the prominent workers who studied the aquatic and wetlands plants of India as a whole. The important works on hydrophytes and aquatic angiosperms in Bangladesh have been carried out by Khan and Mahbuba (1987) and Khan and Halim (1987). Khan and Halim (1987) reported 123 species most of them are well described and illustrated. Kundu (1938), Datta and Mitra (1953), Islam and Paul (1977), Islam et al. (1979), Zaman 
(1991), Khondker et al. (1993), Chowdhury et al. (1996), Yousuf et al. (1997) and Irfanullah (2002) put an emphasis on the relationship between morphological, hydrological, physical and water qualities pattern of wetland system. Aquatic plant distribution has widely been investigated from different parts of country. Besides, several publications like Karim (1993), Hasan (2000) and Rashid (2006) have ventured to study the plant diversity of wetlands of some districts and regions. Islam (2004), Ali (2002), Hossain (2002) and Fatima (2005) worked on Barind tract. Ali and Ahmed (2001) and Rahman et al. (2010) worked on biodiversity conservation and threats on wetlands. Still there is no quantified observation (data) on the hydrophytes in respective of High Barind Tract area in the scientific process and thus their potentiality remains still underutilized. Thus present study aims to identify and assess the hydrophytes diversity in High Barind Tract, observe the scenario of threats of these wetlands which are caused by anthropogenic activities and prepare a strategy of these wetlands conservation by combining indigenous knowledge and findings of the research.

\section{Materials and Methods}

The Kharoil Beel is situated at Mohanpur upzilla under Rajshahi district and at the central region of the High Barind Tract. Mohanpur upzilla is bounded on the North by Manda upzilla of Naogaon zilla, on the East by Bagmara and Durgapur, upzilla on the South by Paba upzilla and on the west by Tanore upzilla. It is located between $24^{\circ} 29^{\prime}$ and $24^{\circ} 38^{\prime}$ north latitude and between $88^{\circ} 34^{\prime}$ and $88^{\circ} 43^{\prime}$ east longitude and has an area of 73.75 acres spreading over 1 mouza and the depth of water varied from 2-4 m. with a maximum reaching up to $5 \mathrm{~m}$. connected the river Bornai which further connected to the river Atrai. The nature of soil in Kharoil beel was moderately alkaline.

The Beel Kumari is situated at Tanore upzilla under Rajshahi district at the central region of the High Barind Tract. Tanore upzilla is bounded in the north by Niamatpur and Manda upzilla, in the south by Paba and Gaodagari, upzilla in the east by Mohanpur upzilla and in the west by Nawabgonj and Nachole upzilla. It lies between $24^{\circ} 29^{\prime}-24^{\circ} 43^{\prime}$ north latitude and $88^{\circ} 24^{\prime}-88^{\circ} 38^{\prime}$ east longitude area of the beel is 502.66 acre spread over Chanduria union to Chowbari union which spreading over 2 mouza. The depth of water varied from 3-5 m with a maximum reaching up to $7-10 \mathrm{~m}$. connected the river river Shib which was further connected to the river Atrai. The nature of soil in Beel Kumari was moderately alkaline.

The Jobai Beel is situated on the Sapahar upzilla under Naogaon district. Sapahar upzilla is bounded in the north by West- Dinajpur of India, in the south by Porsha upzilla, in the east by Potnitola upzilla and in the west by Maldha district of India. It lies between $25^{\circ}$ $01^{\prime}-25^{\circ} 13^{\prime}$ north latitude and $88^{\circ} 26^{\prime}-88^{\circ} 38^{\prime}$ east longitude area of 912.24 acre spreading cover 5 mouzas. The depth of the water varied from $3.5 \mathrm{~m}$ (app.) in dry season, maximum reaching up to 10-12 $\mathrm{m}$ (app.) during rainy season connected the river Dhoala and 
"Purnabhaba" which further connected to the river Atrai. The nature of soil in Jobai beel found slightly acidic to moderately alkaline.

Climate condition of these area experiences with the longest cool winter period (79-100 days) and the highest number of days (5-10 days) with maximum atmospheric temperature above $40^{\circ} \mathrm{C}$ in March-May, whereas the minimum temperature started to fall below $18^{\circ}$ ranges between November. Both in summer and winter seasons the water level of these wetlands gradually fall. Then central portion of these wetland areas proper retains water but greater portion of beels are used for cultivation of crops. June arrives with the monsoon rainfall and the water level of these wetlands begins to increase again; the process continues throughout the entire post monsoon period. During monsoon and post monsoon the three wetlands areas turn into dense aquatic vegetation.

Monthly sampling was made from a period of June 2005 to May 2008 for the collection of hydrophytes plants and to study the diversity status of three wetlands namely Kharoil Beel, Beel Kumari and Jobai Beel. Plant species diversity within the wetlands determined by the quadrate method was used throughout the period of investigation (Krebs 1989). In each three sites, 12 quadrates $(1 \times 1$ acre) were randomly laid to individual number of hydrophytes belonging to each of the species met which was identified and recorded. Hydrophytes species identification was done with the help of standard literatures (Hooker 1872-1897, Roxburgh 1834, Prain 1903, Cook 1996, Kundu 1938, Khan and Halim 1987, Siddique et al. 2007 and Ahmed et al. 2008b, 2009a and 2008-2009). Simpson's diversity index Simpson (1949) and Shanon's diversity index Mac.Arthur (1963) and Peet (1974) were applied to determine species diversity and species richness.

\section{Results and Discussion}

At study areas a total of 151 species and 90 genera distributed in 44 families was identified. For each species scientific name, family and number of individuals of hydrophytes are provided (Table 1). Among the families $65.56 \%$ represented by 12 families and $34.43 \%$ represented by 32 families (Fig. 1). In wetlands plants Azollaceae, Lemnaceae, Nymphaeceae and Menyanthaceae families were found in all study area. The dominant families in order to species richness (more than 5 species) were as follows Cyperaceae with 25 species $>$ Poaceae with 15 species $>$ Scrophulariaceae with14 species $>$ Polygonaceae with 7 species $>$ Commelinaceae with 6 species $>$ and Asteraceae with 5 species. Many of these were not found only to wetland but to nearly moist soil also. Member of Trapaceae was very rare and was found in Mohanpur only. Selaginaceae family found only in Tanore was rare. Salviniaceae family was not found in Sapahar (Table 1). The occurrence and assemblage of macrophytes were because of the affect by abiotic factor such as water quality, sediment properties, temperature and fluctuations in water level etc. 
Table 1. Name of recorded families, species and number of individuals of hydrophytes of study areas.

\begin{tabular}{|c|c|c|c|c|}
\hline \multirow[b]{2}{*}{ Family } & \multirow[b]{2}{*}{ Species Name } & \multicolumn{3}{|c|}{ Locality and number of individuals } \\
\hline & & $\begin{array}{c}\text { Kharoil } \\
\text { Beel }\end{array}$ & $\begin{array}{c}\text { Beel } \\
\text { Kumari }\end{array}$ & Jobai Beel \\
\hline Azollaceae & Azolla pinnata & 2565 & 254 & 2819 \\
\hline \multirow{2}{*}{ Marsileaceae } & Marsilea minuta & - & 11 & - \\
\hline & Marsilea quadrifolia & 2175 & 221 & 211 \\
\hline Adiantaceae & Ceratopteris thalictroides & - & 224 & 101 \\
\hline \multirow[t]{2}{*}{ Salviniaceae } & Salvinia cucullata & 1056 & - & - \\
\hline & Salvinia natans & 1219 & 111 & - \\
\hline \multirow[t]{4}{*}{ Acanthaceae } & Hygrophila auriculata & 211 & - & 217 \\
\hline & Hygrophila difformis & 1219 & 1180 & - \\
\hline & Hygrophila polysperma & 1495 & 1607 & - \\
\hline & Hygrophila quadrivalvis & 17 & - & - \\
\hline Alismataceae & Sagittaria guayanensis & - & 240 & 11 \\
\hline \multirow[t]{3}{*}{ Amaranthaceae } & $\begin{array}{l}\text { Alternanthera } \\
\text { paronychioides }\end{array}$ & 272 & 281 & 222 \\
\hline & $\begin{array}{l}\text { Alternanthera } \\
\text { philoxeroides }\end{array}$ & 481 & 408 & 403 \\
\hline & Alternanthera sessilis & 506 & 439 & 489 \\
\hline \multirow[t]{2}{*}{ Apiaceae } & Centella asiatica & 394 & 40 & 49 \\
\hline & $\begin{array}{l}\text { Hydrocotyle } \\
\text { sibthropioides }\end{array}$ & 16 & - & - \\
\hline \multirow[t]{3}{*}{ Aponogetonaceae } & $\begin{array}{l}\text { Aponogeton } \\
\text { appendiculatus }\end{array}$ & 652 & 236 & - \\
\hline & Aponogeton natans & 82 & - & - \\
\hline & Aponogeton undulatus & 662 & - & - \\
\hline \multirow[t]{3}{*}{ Araceae } & Colocasia antiquorum & 560 & 595 & 536 \\
\hline & Lasia spinosa & 16 & - & - \\
\hline & Pistia stratiotes & 6250 & 6039 & 6898 \\
\hline \multirow[t]{5}{*}{ Asteraceae } & Caesulia axillaris & 898 & 1264 & 1102 \\
\hline & Eclipta alba & 896 & 748 & 811 \\
\hline & Enhydra fluctuans & 1594 & 160 & 113 \\
\hline & Grangea maderaspatina & 1031 & 992 & 734 \\
\hline & Spilanthes acmella & 1069 & 1087 & 728 \\
\hline Balsaminaceae & Hydrocera triflora & $\cdot$ & - & 2 \\
\hline Ceratophyllaceae & Ceratophyllum demersum & - & - & 339 \\
\hline \multirow[t]{6}{*}{ Commelinaceae } & Cyanotis axillaris & - & 732 & 72 \\
\hline & Commelina benghalensis & 1038 & 942 & 1044 \\
\hline & Commelina diffusa & 876 & 83 & - \\
\hline & Floscopa scandens & - & $\therefore$ & 32 \\
\hline & Murdannia nudiflora & 182 & 152 & - \\
\hline & Murdannia spirata & 146 & 191 & 12 \\
\hline \multirow[t]{2}{*}{ Convolvulaceae } & Ipomoea aquatica & 910 & 135 & 824 \\
\hline & Ipomoea fistulosa & 509 & 317 & 490 \\
\hline \multirow[t]{4}{*}{ Cyperaceae } & Carex panicea & 119 & 101 & 104 \\
\hline & Cyperus cephalotes & 579 & 554 & - \\
\hline & Cyperus platystylis & - & 530 & 37 \\
\hline & Cyperus involucratus & - & 32 & 26 \\
\hline
\end{tabular}


Contd.

\begin{tabular}{|c|c|c|c|c|c|}
\hline \multirow[b]{2}{*}{ Family } & \multirow[b]{2}{*}{$\sqrt{2}$} & \multirow[b]{2}{*}{ Species Name } & \multicolumn{3}{|c|}{ Locality and number of individuals } \\
\hline & & & $\begin{array}{c}\text { Kharoil } \\
\text { Beel }\end{array}$ & $\begin{array}{c}\text { Beel } \\
\text { Kumari }\end{array}$ & Jobai Beel \\
\hline & 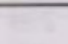 & Cyperus polystachyos & 304 & - & 260 \\
\hline & $\sqrt{2}$ & Cyperus dives & 142 & 29 & 27 \\
\hline & $=$ & Cyperus compressus & 287 & 292 & 275 \\
\hline$y^{1}$ & $=$ & Cyperus kyllingia & 278 & 299 & 255 \\
\hline & $=$ & Cyperus exaltatus & 39 & 547 & $\cdot$ \\
\hline $1 x^{2}$ & 15 & Cyperus difformis & 397 & 378 & 313 \\
\hline 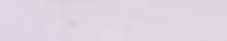 & a & Cyperus iria & 191 & 100 & - \\
\hline & a & Diplacrum caricinum & - & 50 & - \\
\hline$=$ & 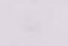 & Eleocharis actangula & 83 & - & - \\
\hline & 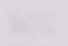 & Elecocharis congesta & - & - & 529 \\
\hline 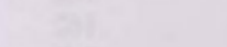 & $x^{2}$ & Eleocharis dulcis & - & 587 & - \\
\hline ay & $2 x$ & Fimbristylis acuminata & 28 & 273 & 28 \\
\hline$\sqrt{2}$ & sin & Fimbristylis aestivalis & 296 & - & 234 \\
\hline & $a_{1}$ & Fimbristylis dichotoma & 592 & - & 333 \\
\hline & 24 & Fimbristylis miliaceae & - & 395 & 312 \\
\hline & 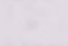 & Mariscus sumatrensis & 189 & - & 20 \\
\hline & 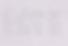 & Scirpus articulatus & 329 & 400 & 303 \\
\hline 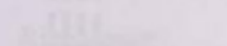 & 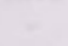 & Scirpus erectus & 153 & - & - \\
\hline & 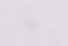 & Scirpus grossus & 162 & 398 & 305 \\
\hline & & Scirpus mucronatus & 73 & - & - \\
\hline & 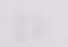 & Scirpus lateriflorus & 34 & 388 & 283 \\
\hline Elatinaceae & $x^{2}$ & Elatine triandra & 884 & 886 & 74 \\
\hline \multirow{2}{*}{ Eriocaulaceae } & ant & Eriocaulon cinereum & 721 & - & - \\
\hline & $2=$ & Eriocaulon setaceum & 303 & - & 172 \\
\hline \multirow[t]{4}{*}{ Fabaceae } & & Aeschynomene aspera & 71 & 7 & 64 \\
\hline & & Aeschynomene indica & - & 66 & 60 \\
\hline & & Sesbania bispinosa & 99 & - & - \\
\hline & & Sesbania roxburghii & - & - & 67 \\
\hline \multirow[t]{2}{*}{ Haloragraceae } & & Myriophyllum indicum & 1375 & - & - \\
\hline & & $\begin{array}{l}\text { Myriophyllum } \\
\text { tuberculatum }\end{array}$ & 1575 & - & - \\
\hline \multirow[t]{7}{*}{ Hydrocharitaceae } & & Blyxa aubertii & 1394 & 146 & - \\
\hline & & $\begin{array}{l}\text { Blyxa abubertii var. } \\
\text { echinosperma }\end{array}$ & - & 20 & - \\
\hline & $=$ & Elodea canadensis & 10 & - & - \\
\hline & & Hydrilla verticillata & 1439 & 144 & 152 \\
\hline & & Nechamandra alternifolia & 1938 & - & - \\
\hline & & Ottelia alismoides & 2624 & - & 268 \\
\hline & . & Vallisneria spiralis & 1678 & - & - \\
\hline Hydrophylaceae & 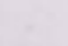 & Hydrolea zeylanica & 306 & - & - \\
\hline Lamiaceae & & Pogastemon stellatus & 310 & - & - \\
\hline \multirow[t]{2}{*}{ Lemnaceae } & & Lemma perpusilla & 3414 & 3350 & 3281 \\
\hline & & Spirodela polyrhiza & 2800 & 4170 & 2327 \\
\hline \multirow[t]{3}{*}{ Lentibulariacae } & & Utricularia aurea & 3111 & - & - \\
\hline & & Uiricularia exoleta & - & 306 & - \\
\hline & & Utricularia inflexa & - & - & 32 \\
\hline
\end{tabular}


Contd.

\begin{tabular}{|c|c|c|c|c|c|}
\hline \multirow[b]{2}{*}{ Family } & \multirow{2}{*}{\multicolumn{2}{|c|}{ Species Name }} & \multicolumn{3}{|c|}{ Locality and number of individuals } \\
\hline & & & $\begin{array}{c}\text { Kharoil } \\
\text { Beel }\end{array}$ & $\begin{array}{c}\text { Beel } \\
\text { Kumari }\end{array}$ & Jobai Beel \\
\hline \multirow{5}{*}{ Lythraceae } & 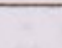 & Utricularia stellaris & 2806 & 299 & - \\
\hline & 68 & Ammannia baccifera & 27 & 25 & 26 \\
\hline & 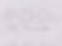 & Rotala indica & 209 & - & - \\
\hline & & Rotala rotundifolia & 208 & - & 76 \\
\hline & $x^{2}$ & Rotala wallichii & - & 5 & - \\
\hline \multirow[t]{2}{*}{ Menyanthaceae } & a & Nymphoides indica & 1694 & 166 & 220 \\
\hline & $(14$ & Nymphoides cristatum & 144 & 138 & $\cdot$ \\
\hline \multirow[t]{2}{*}{ Najadaceae } & y & Najas graminea & 1293 & - & - \\
\hline & & Najas minor & 827 & - & - \\
\hline \multirow[t]{4}{*}{ Nymphaeceae } & 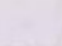 & Nymphaea nouchali & 2033 & 204 & 162 \\
\hline & & Nymphaea rubra & 1811 & 198 & 162 \\
\hline & & Nymphaea stellata & - & 185 & 162 \\
\hline & & Euryale ferox & - & - & 23 \\
\hline Onagraceae & & Ludwigia adscendens & 865 & 881 & 89 \\
\hline \multirow[t]{15}{*}{ Poaceae } & & Coix aquatica & 99 & 119 & - \\
\hline & & Coix lacryma-jobi & - & - & 76 \\
\hline & 15 & Echinochloa colona & 1558 & 1721 & 1272 \\
\hline & 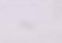 & Echinochloa crus-galli & 1178 & - & 1111 \\
\hline & 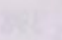 & Eragrostis uniloides & - & - & 75 \\
\hline & $=$ & Hygroryza aristata & 91 & - & 83 \\
\hline & $c^{2}$ & Hymenachne acutigluma & 108 & 42 & 80 \\
\hline & in & Leersia hexandra & 144 & 136 & 146 \\
\hline & $=$ & Leptochloa chinensis & 129 & 109 & 112 \\
\hline & 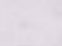 & Oryza rufipogon & 144 & 128 & 123 \\
\hline & 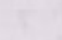 & Panicum paludosum & 10 & 28 & 112 \\
\hline & 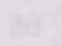 & Paspalum paspaloides & 119 & 99 & 103 \\
\hline & 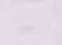 & Phragmites karka & - & - & 121 \\
\hline & = & Saccharum spontaneum & 169 & 138 & 141 \\
\hline & 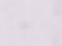 & Vetiveria zizannioides & 130 & 114 & 113 \\
\hline \multirow[t]{7}{*}{ Polygonaceae } & 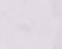 & Persicaria orientale & 68 & 382 & 409 \\
\hline & & Persicaria barbatum & - & 53 & - \\
\hline & 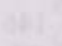 & Persicaria glabrum & - & 51 & - \\
\hline & . & Persicaria hydropiper & 64 & - & - \\
\hline & & Polygonum plebejum & 62 & - & - \\
\hline & . & Persicaria tomentosum & 56 & - & 371 \\
\hline & $\sqrt{1}$ & Rumex martimus & 40 & 160 & - \\
\hline \multirow[t]{3}{*}{ Pontederiaceae } & 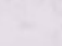 & Eichhornia crassipes & 1143 & 1152 & 1068 \\
\hline & 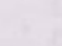 & Monochoria hastata & 1479 & 995 & 80 \\
\hline & $=$ & Monochoria vaginalis & - & - & 40 \\
\hline \multirow[t]{2}{*}{ Potamogetonaceae } & 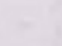 & Potamogeton crispus & 76 & - & - \\
\hline & & Potamogeton nodosus & - & 32 & - \\
\hline \multirow[t]{2}{*}{ Ranunculaceae } & & Ranunculus sceleratus & 125 & 122 & 102 \\
\hline & & Dentella repens & 232 & 23 & 17 \\
\hline & & Oldenlandia corymbosa & 226 & 219 & - \\
\hline \multirow{2}{*}{ Scrophulariaceae } & 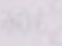 & Bacopa monniera & - & 229 & - \\
\hline & - & Dopatrium junceum & 222 & - & 187 \\
\hline
\end{tabular}


Contd.

\begin{tabular}{|c|c|c|c|c|}
\hline \multirow[b]{2}{*}{ Family } & \multirow[b]{2}{*}{ Species Name } & \multicolumn{3}{|c|}{ Locality and number of individuals } \\
\hline & & $\begin{array}{c}\text { Kharoil } \\
\text { Beel }\end{array}$ & $\begin{array}{c}\text { Beel } \\
\text { Kumari }\end{array}$ & Jobai Beel \\
\hline & Limnophila aromatica & 119 & 128 & 120 \\
\hline & Limnophila chinensis & 62 & - & - \\
\hline & Limnophila cana & 7 & - & $\cdot$ \\
\hline & Limnophila heterophylla & 1 & - & 112 \\
\hline & Limnophila indica & 1167 & - & - \\
\hline & Limnophila polystachya & 654 & - & - \\
\hline & Limnophila sessiliflora & 548 & - & - \\
\hline & Lindernia antipoda & 190 & 212 & 193 \\
\hline & Lindernia crustacea & 210 & 230 & 228 \\
\hline & Lindernia ciliata & - & 248 & - \\
\hline & Lindernia rotundifolia & - & - & 199 \\
\hline & $\begin{array}{l}\text { Veronica anagallis- } \\
\text { aquatica }\end{array}$ & - & 114 & 12 \\
\hline Trapaceae & Trapa bispinosa & 111 & - & - \\
\hline Verbanaceae & Phyla nodiflora & 191 & 190 & 193 \\
\hline \multirow[t]{2}{*}{ Characeae } & Chara corallina & 1013 & 101 & 706 \\
\hline & Nitella furcata & 1125 & 113 & 674 \\
\hline Ricciaceae & Riccia sp. & 148 & - & - \\
\hline Selaginaceae & Selaginella sp. & - & 26 & - \\
\hline Boraginaceae & Heliotropium indicum & - & 40 & - \\
\hline Campanulaceae & Lobelia zeylanica & 55 & - & - \\
\hline
\end{tabular}

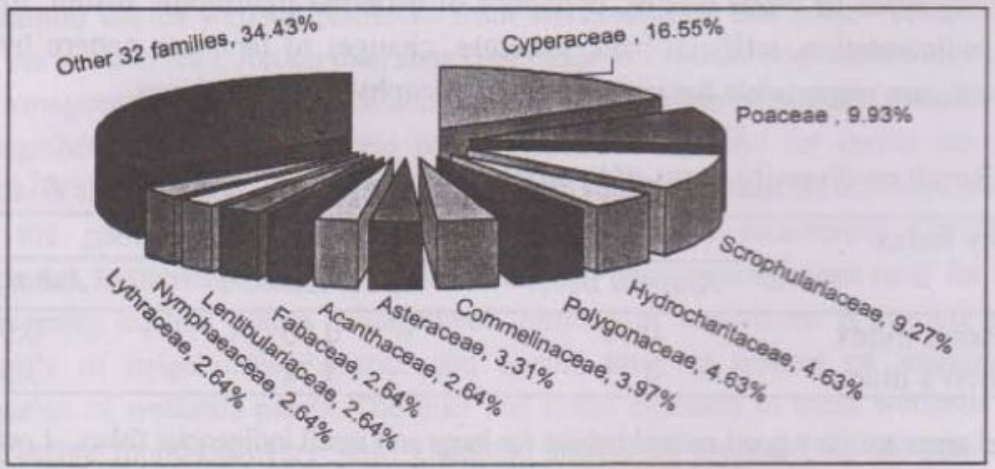

Fig.1. Distribution of species in the families.

From the Table 2 it is evedent that at Kharoil Beel a total of 117 species under 75 genera distributed in 38 families were present. Among these the largest number of species (19) belonging to the family Cyperaceae followed by Poaceae and Scrophulariaceae. Azollaceae, Elatinaceae, Hydrophyllaceae, Lamiaceae, Onagraceae, Verbanaceae, Ricciaceae, Rubiaceae, Campanulaceae and Trapaceae are represented by a single species. The other families had a varying number of species ranging from 2-10 (Table 1). 
In Beel Kumari, a total of 92 species under 65 genera distributed in 34 families was identified (Table 2). Maximum number (17) of species were reported to be the member of family Cyperaceae followed by Poaceae (10). Azollaceae, Adiantaceae, Alismataceae, Boraginaceae, Selaginaceae, Verbanaceae and Rubiaceae are represented by a single species. Rest of the families had a varying number of species ranging from 2-7 (Table 1).

Table 2. Comparative analysis of species distribution of study areas.

\begin{tabular}{cccc}
\hline Name of beels & Family & Genus & Species \\
\hline Kharoil Beel & 38 & 75 & 117 \\
Beel Kumari & 34 & 65 & 92 \\
Jobai Beel & 33 & 70 & 90 \\
\hline
\end{tabular}

In Jobai Beel, a total of 90 species under 70 genera distributed in 33 families have been identified (Table 2). Maximum number of species (17) belonged to the family of Cyperaceae followed by Poaceae represented by (14) species. Azollaceae, Elatinaceae, Onagraceae, Alismataceae, Balsaminaceae, Verbanaceae, Adiantaceae, and Ceratophyllaceae are represented by a single species. The other families had a varying number of species ranging from 2-7 (Table 1).

Result suggests that hydrophytes diversity of Kharoil Beel was 0.97 in Simpson's index and 4.13 in Shannon's index (Table 3). In Beel Kumari was 0.95 in Simpson's index and 3.71 in Shannon's index (Table 3). Observation on Jobai Beel was 0.94 in Simpson's index and 3.55 in Shannon's index (Table 3). All of them show rich diversity. Connectivity types of water bodies, dynamics of different hydrologic matter, high flow velocity sedimentation, artificial bank structure, changes of land use pattern by human pressure etc., are responsible for variability of hydrophytes assemblages.

Table 3. Result on diversity status of hydrophytes.

\begin{tabular}{lccc}
\hline Diversity index & \multicolumn{3}{c}{ Name of the beel areas } \\
\cline { 2 - 4 } & Kharoil Beel & Beel Kumari & Jobai Beel \\
\hline Simpson's index & 0.97 & 0.95 & 0.94 \\
Shannon's index & 4.13 & 3.71 & 3.55 \\
\hline
\end{tabular}

These beel areas are very good natural habitat for large and small indigenous fishes. Local people are engaged in catching fish for house hold consumption. In summer and winter season, greater part of these beel are converted to agriculture. In dry season the influential persons capture the beel land illegally, though these are government managed jolmahal .Besides this, a large number of economical and ecological wetland plants are used by villagers. These are Thankuni (Centella Asiatic), Kulekhara (Hygrophilla sp.), Eclipta alba, Hingcha (Enydra Fluctuans), Alternanthera sessilis (Sachishak), Alternanthera philoxeroides (Helencha), Ipomoea aquatica (Kalmi sak), Colocosia antiquorum (Kachu), Centella asiatica (Thankuni), Enhydra fluctuans (Helencha), Trapa bispinosa (Paniphal) and Euryale ferox (Makhna)etc. 
The villagers collect them and sell them to the local market, thus supplement their household economy upturn.

The livelihoods of the people of surrounding area are mostly depended on this wetland resource (Kostori M.F.A. 2012). Consequently, the human activities in the studied area in fact mainly led to an increase in its hydrophytes diversity. During the field observation and discussion with local people it was identified that a number of threats happened due to human interventions, through conversion of wetland to agriculture land, indiscriminate use of pesticide and insecticides, pollution by domestic waste products, conversion of wetland for development work as well as land scape alteration, extensive fishing, unsustainable use of water resources, land grabbing of local people etc. led to a severe decrease in the diversity of aquatic plants of these reported areas. It was further noticed that local people tend to destroy the wetland plants as well as wetlands resources for their demands due to lack of awareness, proper intuitional coordination and management frame work and monitoring. As a result, many species of hydrophytes like Euryale ferox, Hydrocera triflora, Oryza rufipogon, Trapa bispinosa etc. and fauna are threatened. Wetland based ecosystems degenerating as well as livelihood pattern, socio -economic framework and cultural values are affected. In addition government is losing huge sum of revenues since these are khas land.

These wetlands aquatic system that support a wide range of aquatic plant diversity for maintaining ecological balance with their own scenic beauty, provide indispensable benefits to the human livelihood, their socio-economic system. The recommendations have been made for the sustainable conservation of hydrophytes diversity in these wetlands. Awareness, motivation and ensuring best practices among local people are necessary about wetland value and optimum use of wetland resources from environmental and socio- economic view to protect the wetland area. Action plan should be made to facilitate coordinated and cooperative based management approach. Local community and all levels of relevant personnel's should work together in the entire process of implementation phases for conservation of these wetlands. With the help of appropriate instruments, structures, realistic activities are needed to ensure the good governance in wetlands management, monitoring and community development. Initiatives should be taken for "aquatic plants management unit" for integration on concerning aquatic plants management. Sharing of experience is needed among the personnel's of neighbouring district and upzilla level in respect of management and conservation of wetlands plants. The inlet and outlet channels to these wetlands should be cleared before monsoon every year. Database should be prepared for all plant species in respective wet land areas. Conservation should be ensured and maintained by talking different activities such as plantation programme with indigenous species and maintaining close season, protecting harmful gear, such as jal, putijal, traps etc., demonstrating of eco friendly agriculture, utilization of hydrophytes, controlling these plants cheaply, restoring of water bodies to their intended uses and protect liberating encroached. Jolmahal leasing system should be phased out in favour of pro-poor community who will ensure conservation and management of these wetlands resources specially on account of aquatic plants in order to respective policy, rules and legal frame work. 
Based on the field observations and present preliminary results, it may be concluded that study sites are rich in the hydrophytes diversity which however, is severely threatened because of anthropogenic pressure. Therefore, in the context of Bangladesh many hydrophytes species will be extinct before their introduction to science. In the prevailing circumstances, awareness building, motivation and best practice among local people about focusing all aspects of hydrophytes diversity of these wetlands could be acted for reaching the destination in protecting the wetland areas and strengthening their plant conservation. Finally, it appears that there is a scope for long term research on these wetlands regarding the conservation and management of their plant diversity.

\section{References}

Ahmed, Z.U., Z.N.T. Begum, M.A. Hasan, M. Khondker, S.M.H. Kabir, M. Ahmed, A.T.A. Ahmed, A.K.A Rahman and E.U. Haque (Eds.) 2008-2009. Encyclopedia of Flora and Fauna of Bangladesh. Vols.6-10. Angiosperms: Dicotyledons, Asiatic Society of Bangladesh, Dhaka.

Ahmed, Z.U., M.A. Hasan, Z.N.T. Begum, M. Khondker, S.M.H. Kabir, M. Ahmed, A.T.A. Ahmed, A.K.A Rahman and E.U. Haque (Eds.) 2008b. Encyclopedia of Flora and Fauna of Bangladesh. Vol.12. Angiosperms: Monocotyledons. Asiatic Society of Bangladesh, Dhaka. pp.1-552.

Ahmed, Z.U., M.A. Hasan, Z.N.T. Begum, M. Khondker, S.M.H. Kabir, M. Ahmed, A.T.A. Ahmed, A.K.A Rahman and E.U. Haque (Eds.) 2009a. Encyclopedia of Flora and Fauna of Bangladesh. 7: Angiosperms: Dicotyledons, Asiatic Society of Bangladesh, Dhaka pp.1-546.

Ali, M.O. and M. Ahmed. 2001. Bio-diversity conservation: vision for Bangladesh. In: Chowdhury, Q.I.(Ed). Bangladesh: State of Biodiversity.Forum of Environmental Journalists of Bangladesh (FEJB), Dhaka.33-49 pp

Ali, Md. Showkat. 2002. Hydrobiological Characteristics of Barind tract: a case study of Tanore Thana. M. phil. Thesis. Dept of Geography and Environmental studies. University of Rajshahi. pp.133.

Biswas, K. and C.C. Calder. 1954. (Reprinted 1984). Hand book of common water and marsh plants of India and Burma. Bishen Singh Mahendra Pal Singh, Dehra Dun, India, pp. 329.

Chowdhury, A.H., S. Naz., and M. Zaman. 1996. Effect of industrial effluents on the physicochemical and biological conditions of Padma water near Rajshahi. J. Bio. Sci. 4:9-19

Cook, C.D.K. 1996. Aquatic and Wetland Plants of India. Oxford University Press. United Kingdom. pp. 1-385.

Datta, R.M. and J. N. Mitra. 1953. Common plants in and around Dacca. Bull. Bot. Soc. Beng. 7(1 and 2$): 47-48$.

Dugan, P.J. (ed.) 1990. Wetland Conservation: A Review of Current Issues and Required Action. IUCN, Gland, Switzerland. p. 305.

Fatima, Kanij Sultana. 2005 .Phytoplankton of Barind waters in relation of physico-chemical conditions. Ph.d thesis. Dept. of Botany, University of Rajshahi. pp. 448.

Hasan, M. A. 2000. Assessment of diversity of algal plants in Chalan Beel in relation to physicochemical conditions, Ph. D thesis, Dept. of Botany, Rajshahi University. pp. 420.

Hooker J.D.1872-1897. The Flora of British India. Vols.1-7. M.S. Bishen Singh Mehedra Pal Singh, Dehra Dun and M.S. Periodical Experts. Delhi (Indian reprint 1973). 
Hossain, M.A. 2002. Production system, productivity and its augmentation of Altadighi, Dhamairhat in the district of Naogaon. Ph.D., thesis. Dept. of Zoology. Rajshahi University. pp. 224.

Irfanullah, H. M. 2002. Studies on aquatic vascular plants in Bangladesh: An Appraisal, Bangladesh J. Plant taxon. Vol. 9 (1): 85-116.

Islam, Md. Azizul. 2004. Investigation on the plankton biota of Barind wetland and Mohanpur in relation to its physicochemical condition. Ph.D Thesis, Dept. of Botany, University of Rajshahi.pp. 163.

Islam, A.K.M. N. and S.N. Paul. 1977. Limnological studies on Wolffia arrhiza (L.) Wiom. J. Asiatic Soc. Bangladesh Sci. Vol. 3(1): 111-123.

Islam, A.K.M.N., M. Rahman and A.R. Chowdhury. 1979. Hydrobiological studies of Dhanmondi Lake, Dacca. 1. Macrophytes and benthic flora. J. Asiatic Soc. Bangladesh Sci. Vol. 5 (1): 59-75.

Karim ,A. 1993. Plant Diversity and their Conservation in Freshwater Wetlands, In : Nishat A, Z. Hussain, M.K. Roy and A. Karim (eds.). Freshwater Wellands in Bangladesh: Issues and Approaches for Management. IUCN, The World Conservation Union. pp. 75-104.

Khan ,M.S. and Mahbuba. H.M. 1987. Aquuatic angiosperm of Bangladesh. National Herbarium of Bangladesh. 1-4: 1-120.

Khan ,M.S and M. Halim. 1987. Aquatic angiosperms of Bangladesh national Herbarium BARC, pp. 114.

Khan, H.R. 1993. Water Development Aestivates and their Impacts on Wetlands. In: A. Nishat, Z. Hossain, Roy, M. K. and Karim, A. (eds.). Fresh. pp. 23-32.

Khondker, M., A.K.M.N. Islam and A.D. Makhnun. 1993. Study of the growth of a floating macrophyte. J. Asiatic Soc. Bangladesh. Sci. 19 (2): 103-108.

Krebs, C.J. 1989. Ecological Methodology. Harper Collins Publishers, 654pp.

Kundu, B.C. 1938. A. new species of Nitella from Rajshahi, Bengal J. India Bot. Soc. 16: 223-226.

Kostori ,M. F.A. 2012 .Socio-economic Condition of Fishermen of the Chalan Beel underTarash Thana of Sirajganj District in Bangladesh. Bangladesh Research Publications Journal, Vol. 6, Issue 4, March-April,2012, pp. 393-402.

Mac Arthur, R.H.1963. Patterns of species diversity. Biol. Rev, 40:510-533.

Peet, R.K. 1974. The measurement of species diversity. Ann. Rev. Eco. and Syst. 5: 285-307.

Prain, D. 1903. Bengal Plants. Botanical Survey of India, Calcutta. Vols. I \& II. Bishen Singh Mahendra Pal Singh, Dehra Dun, India, (Reprinted, 1963). pp. 1013.

Roxburgh, W. 1834. Flora Indica, Cronica Botanica. pp. 795.

Rahman, M.M., Rahman M. R and Asaduzzaman M. 2010. The Aggression of Human Activitieson Chalan Beel A Threat on Wetland Environment: Study on Natore-Rajshahi Regionof Bangladesh. Journal of Science Foundation, 8:(1-2) 151-159.

Rashid,Md.Abdur.2006. Ecology and Floristic diversity of hydrophytes in Chalan beel wetlands.Ph.D.thesis. Dept. of Botany.University of Rajshahi.pp.356.

Siddique, K.U., Islam, M.A., Ahmed, Z.U., Begum, Z.N.T., Hassan, M.A., Khondker, M., Rahman, M.M., Kabir, S.M.H., Ahmad, M., Ahmed, A.T.A., Rahman, A.K.A. and Haque, E.U. (Eds) 2007. Encyclopedia of Flora and Fauna of Bangladesh, 11. Angiosperms: Monocotyledons. Asiatic Society of Bangladesh, Dhaka, pp. 1-399.

Simpson, E.H. 1949. Measurement of diversity. Nature. 163:688.

Subramanyam, K.1962. Aquatic angiosperms, Indian Council of Scientific and Industrial Research, New Delhi. pp. 190. 
Yousuf, M., M.O. Rahman, M.S. Khan and S. Huq.1997.Angiospermic flora of Chanda Beel, Gopalgonj district in Bangladesh J.Plant Taxon. 4(2):25-36.

Zaman, M. 1991. Studies on the algal flora of Chalan beel in relation to its Physico-chemical conditions. Ph. D. thesis, Dept. of Botany, University of Rajshahi, Bangladesh. pp. 411.

(Received revised manuscript on 20 November 2014) 